\title{
Caries Process on Occlusal Surfaces: Evolving Evidence and Understanding
}

\author{
J.C. Carvalho \\ School of Medicine and Dentistry, Catholic University of Louvain, Brussels, Belgium
}

\author{
Key Words \\ Active lesions $\cdot$ Arrested lesions $\cdot$ Dental biofilm $\cdot$ Occlusal \\ caries $\cdot$ Tooth eruption
}

\begin{abstract}
Management of the caries process on occlusal surfaces of permanent molars has proven a major challenge. The onset of caries on these surfaces takes place soon after their eruption, and the permanent first molars, followed by the second molars, remain the sites in the dentition which show the highest caries prevalence. This paper is structured in the form of questions and answers in which traditional concepts of caries susceptibility of occlusal surfaces are appraised and confronted with the current evidence. Then, research studies examining the role of biological determinants on the development and arrest of occlusal caries in young permanent teeth are discussed. Finally, the contribution of these studies in terms of developing the available scientific evidence and our understanding of the caries process on occlusal surfaces is analyzed. The current evidence does not support the concept that the early onset and high prevalence of occlusal caries in young permanent teeth are due to a particularly low inherent resistance of the occlusal surface or due to the presence of inaccessible fissure-like structures on these surfaces. Evidence is provided to show that the most influential biological determinants of the development and arrest of occlusal caries are thick plaque accumulation on the groovefossa system and the stage of tooth eruption limiting mechanical oral function. Consequently, active occlusal lesions
\end{abstract}

are significantly more prevalent in erupting than in fully erupted teeth. The major contribution of this review is to provide updated knowledge about the biological principles determining the development and arrest of caries on occlusal surfaces of erupting teeth.

(c) 2014 S. Karger AG, Basel

In the last 50 years, the combination of intensive laboratory and clinical research in the field of cariology and the observed improvement in oral heath in different populations has provided a deeper understanding of the initiation, development and arrest of the caries process at subclinical and clinical levels [Thylstrup and Fejerskov, 1994; Thylstrup et al., 1994; Carvalho et al., 2004; Marsh, 2004; Mejàre et al., 2004; Nyvad, 2004; ten Cate et al., 2008]. Dental caries is a process resulting from complex interactions between microbial deposits and oral fluids in relation to subsequent changes in dental hard tissues [Fejerskov and Thylstrup, 1994]. This process may take place on any tooth surface where metabolically active microbial deposits, i.e. dental biofilm, are allowed to accumulate and mature over a period of time. It is important to appreciate that the presence of dental biofilm itself does not necessarily result in the development of caries lesions which can be clinically and/or radiographically identified [Carvalho et al., 2004]. In order words, whenever the acid produced by bacteria is neutralized by saliva or other metabolic events in the dental biofilm, tooth demineralization and remineralization

\section{KARGER}

E-Mail karger@karger.com

www.karger.com/cre
(C) 2014 S. Karger AG, Basel

0008-6568/14/0484-0339\$39.50/0
Joana Carvalho

School of Medicine and Dentistry, Catholic University of Louvain

Avenue Hippocrate 10

BE-1200 Brussels (Belgium)

E-Mail joana.carvalho@uclouvain.be 
may compensate one another [Takahashi and Nyvad, 2008]. Such episodes are observed in low cariogenic challenges like those produced by the metabolism of salivary glycoproteins or of fermentable carbohydrates consumed at low frequency.

However, with increased consumption of fermentable carbohydrates, acid production is more intense and the events of demineralization may no longer be compensated by remineralization, resulting in a net loss of minerals from the tooth. The signs of enamel porosity can be identified at subclinical or clinical levels, depending on the extent and frequency of the cariogenic challenges.

Currently, dental caries is considered a natural process as the formation of dental biofilm and its metabolic activity cannot be prevented [Baelum et al., 2008]. Nevertheless, caries progression may be controlled in such a way that visible lesions at clinical and/or radiographic levels never form. Achieving this is probably the main challenge to the dental professional perspective on oral health promotion in 21 st-century populations.

Recently, it has been suggested that the control of oral conditions such as the caries process should be based on management models of chronic diseases, in which multiple strategies are applied to target determinants at individual, family and community levels [Ismail et al., 2009]. While nonbiological determinants have increasingly been studied for their influence on oral health [Mattila et al., 2005; Watt, 2007; Carvalho et al., 2009a, b; Ismail et al., 2009; Skeie et al., 2010; Fontana et al., 2011], the role of biological determinants is of the utmost importance to control caries progression at the level of populations and individuals. Management of the caries process still remains the foremost problem in daily practice for general practitioners as well as for the international scientific community. The major challenge in the management of the caries process is to control caries progression mainly by non-operative treatments and by limiting the number of individuals in a population subject to operative treatments.

Particularly the management of occlusal caries on permanent molars has proven a great challenge, as the onset of caries takes place soon after their eruption into the oral cavity [Carvalho et al., 1989; Ekstrand et al., 2000; Maltz et al., 2003; Ferreira Zandoná et al., 2012]. In spite of the remarkable decline in caries observed since the 1970s in different countries and populations, the occlusal surfaces of permanent first molars, followed by the second molars, remain the sites in the dentition which are most frequently attacked by dental caries [Carvalho et al., 2001; Marthaler, 2004].
This paper is structured in the form of questions and answers in which traditional concepts of caries susceptibility of occlusal surfaces are appraised and confronted with the current evidence. Then, research studies examining the role of biological determinants for the development and arrest of occlusal caries in young permanent teeth are discussed. The discussion of these research studies is presented in the form of hypotheses which were tested. Finally, the contribution of these studies in terms of developing the available scientific evidence and our understanding of the caries process on occlusal surface is analyzed.

\section{How Does One Explain the Early Onset and the High Prevalence of Occlusal Caries?}

Traditionally, occlusal surfaces have been regarded as susceptible to dental caries as a result of their incomplete posteruptive maturation, i.e. their reduced mineral content in the enamel [Kotsanos and Darling, 1991; Schulte et al., 1999; Kataoka et al., 2007], and due to the presence of narrow and deep fissures on the occlusal surfaces which harbor bacteria that initiate the caries process [MullerBolla et al., 2009; Hevinga, 2011; Kühnisch et al., 2012; Liu et al., 2012; Wang et al., 2012]. In order to appreciate how incomplete posteruptive maturation and the presence of deep fissures could be related to the early onset and high prevalence of caries on occlusal surfaces, further explanation is warranted.

\section{What Is the Underlying Rationale for the Concept of Incomplete Posteruptive Maturation?}

The concept of immature teeth is based, on the one hand, on the observation that porosity has been identified in the enamel of erupting teeth and, on the other hand, on the fact that an increased fluoride concentration has been identified in surface enamel after eruption. This observation suggested by analogy that enamel undergoes a period of posteruptive maturation following tooth eruption [Kotsanos and Darling, 1991; Schulte et al., 1999; Kataoka et al., 2007]. A mechanism of action in the maturation process mediated by neutral $\mathrm{pH}$ conditions has never been explained - in particular, how the fluoride would be incorporated into the enamel. In fact, a maturation process, understood in the traditional way, is unlikely to take place. A more logical explanation is that during the eruption period, the enamel surface is undergoing 
several cycles of de- and remineralization, and fluoride present in the oral environment is leading to a gradual increase in fluoride in the outermost part of the surface enamel [Fejerskov et al., 1981; Fejerskov, 2004; Wang et al., 2005; ten Cate et al., 2008]. When the enamel is exposed to low concentrations of ionic fluoride in an acid environment, fluorapatite is formed and deposited as part of the enamel tissue [Tenuta and Cury, 2010; ten Cate, 2013]. It should, however, be appreciated that the overall substitution of hydroxyl ions by fluoride on surface enamel is limited, and that fluoride in the enamel plays a minor role in caries reduction [Fejerskov et al., 1981; Aoba and Fejerskov, 2002].

As a rule, when a tooth erupts into the oral cavity, the enamel is fully mineralized. A certain percentage of teeth may present qualitative and/or quantitative hypomineralization such as non-fluorotic opacities, fluorotic opacities or hypoplasia. Non-fluorotic opacities and fluorotic opacities are not associated with an increased risk of caries development [Carvalho et al., 2011]. Hypoplasia may cause concerns regarding the risk of caries, tooth sensitivity, the placing of restorations and esthetics; however, a large population-based study showed that the prevalence of molar-incisor hypomineralisation in the permanent dentition was only $8.7 \%$ at child level and $5.4 \%$ at tooth level [Elfrink et al., 2012]. On this basis, the early onset of caries on occlusal surfaces and its high prevalence should not be explained by the concept of erupting teeth being immature and thus more prone to caries.

\section{What Is the Role of Narrow and Deep Fissures in Occlusal Caries Development?}

For dental plaque to be cariogenic, i.e. to produce quantities of organic acids sufficient to dissolve the enamel, a protected environment is needed. In the oral cavity, protected environments are found in the so-called 'stagnation areas' such as the cervical third of smooth and interproximal surfaces as well as on occlusal surfaces [Thylstrup et al., 1994]. These stagnation areas are protected from mechanical oral function, which comprises mastication and muscular movements of the tongue, lips and cheeks as well as those involved in the swallowing reflex. On occlusal surfaces, most attention has been devoted to stagnation of dental biofilm in fissure-like structures and the possible events taking place in their deepest part.

Few studies investigated the contribution of the macromorphology of the groove-fossa system to the develop- ment of caries on occlusal surfaces [Thylstrup et al., 1989; Ekstrand and Bjørndal, 1997; Pearce et al., 1999]. The groove-fossa system is defined as the system that connects every single groove and fossa on the occlusal surface. A 'fossa' is a depression on an occlusal surface where two or more grooves meet. An 'interlobal groove' is a groove located between two or more lobes. An 'intersegmental groove' is a groove located between two lobe segments. Together, the fossae and the interlobal and intersegmental grooves are referred to as 'anatomical sites' of the groove-fossa system. These fundamental macroscopic units are considered positive structures which may well present negative structures that have a fissure-like morphology [Carlsen, 1987]. Interlobal grooves with structure angles equal to or less than $25^{\circ}$ are considered as having a fissure-like morphology [Ekstrand and Bjørndal, 1997]. In this paper, the terminology 'narrow and inaccessible fissures' will henceforth be replaced by 'fissurelike structures', as recommended by Carlsen [1987] and Ekstrand and Bjørndal [1997].

In order to improve our knowledge about the role of fissure-like structures in caries development on occlusal surfaces, the correlation between morphology of the groove-fossa system, plaque accumulation and caries development on occlusal surfaces was investigated in a clinical study. The first hypothesis to be tested was the following.

\section{Hypothesis 1:}

The Anatomical Sites of the Groove-Fossa System with Inaccessible Fissure-Like Structures Accumulate

More Plaque and Consequently Present More Carious

Lesions than Morphologically Accessible Sites

In this clinical study [Carvalho et al., 1989], two independent examiners recorded the occurrence and distribution of plaque as well as the localization of caries lesions on occlusal surfaces of permanent first molars in 6- to 8-year-old children. Mouth impression with silicone putty was taken and stone models were prepared. The models were used for a simple visual classification of the macromorphology of the anatomical sites and to verify whether the antagonizing first molars were partly erupted or in full occlusion. The individual anatomical sites were classified as either accessible or inaccessible. If the bottom of the individual anatomic sites was clinically visible, they were classified as accessible, whereas if the bottom of these sites was not visible with the naked eye, they were recorded as inaccessible. A clear association between the visual diagnosis of inaccessibility of the groove-fossa system with plaque accumulation and dental caries was not 
shown. However, occlusal caries and plaque accumulation in specific anatomical sites were closely related $(\mathrm{p}<$ 0.001 ). Since the results showed that the identification of caries-susceptible sites on occlusal surfaces should be based on actual plaque accumulation rather than on the presence of fissure-like structures, the hypothesis tested could not be accepted.

Further, the clinical findings were supported by the natural history of occlusal caries [Thylstrup et al., 1989]. In order to explore the natural interplay between the use of teeth and occlusal caries, the authors considered it necessary to study extracted teeth from a country like Zimbabwe with limited oral health care. Teeth with increasing surface wear and sparse bacterial deposits and teeth with little wear and different degrees of undisturbed bacterial deposits and carious destruction were examined. The teeth were serially sectioned and the sections examined by stereopolarized light microscopes. Teeth from the group with increasing surface wear and sparse bacterial deposits showed different stages of arrested occlusal caries irrespective of fissure-like structure depth. The anatomical sites of the groove-fossa system associated with arrested lesions presented fissure-like structures, most often completely filled with calculus as identified in polarized light. The combined clinical and microscopic examination of teeth with little wear and different degrees of undisturbed bacterial deposits and carious destructions indicated that occlusal enamel breakdown was always related to the deepest or most protected parts of the surface profile.

Moreover, ultrastructural studies indicated that the bacteria at the deepest part of the fissure-like structures were not viable or even calcified, while at the entrance of these fissure-like structures, the bacteria showed intense metabolic activity characterized by multiple cell divisions with morphologically intact microorganisms. Further, it was shown that fissure-like interlobal grooves were not more prone to caries than groove-like interlobal grooves [Ekstrand and Bjørndal, 1997]. These findings indicated that the macromorphology of the occlusal surface, and not the fissure-like structures per se, had a major influence on plaque accumulation and further enamel demineralization.

Accordingly, the early onset and high prevalence of occlusal caries in young permanent teeth could be explained neither by a particularly low inherent resistance of the occlusal surface, focusing on enamel mineralization, nor by the presence of inaccessible fissure-like structures. However, additional evidence about biological determinants associated with occlusal caries was warranted in order to provide a better understanding of the onset and development of caries on occlusal surfaces of newly erupted teeth. In this context, the influence of the eruption stage on occlusal plaque formation and caries development had never been investigated. It was considered relevant to explore these biological phenomena as described in the next section.

\section{Which Are the Biological Determinants of Occlusal Caries Development and Arrest?}

To answer this question, four hypotheses (hypotheses $2-5$ ) were sequentially put forward and tested in a series of three clinical trials designed for children with permanent first molars at different stages of eruption [Carvalho et al., 1989, 1991, 1992]. To test these hypotheses, the clinical examinations recorded: (1) the stage of eruption of permanent first molars; (2) the occurrence and distribution of occlusal plaque in the groove-fossa system; and (3) the occurrence and localization of occlusal caries in the groove-fossa system supplemented with bitewing radiographs.

\section{Hypothesis 2:}

The Interaction between Occlusal Surface-Specific

Macromorphology, Stage of Eruption and Mechanical

Oral Function Determines the Distribution of Dental

Plaque on Occlusal Surfaces

Permanent first molars of the children participating in a clinical trial [Carvalho et al., 1989] were classified according to their stage of eruption, and two indices were developed for the assessment of occlusal plaque. The first index, called 'visible occlusal plaque', described the occurrence and accumulation of occlusal plaque independently of its localization in the groove-fossa system. The second index, called 'detailed mapping of occlusal plaque', described the occurrence, accumulation and localization of the occlusal plaque according to the individual anatomical sites. All recordings were mapped on morphology cards illustrating the occlusal surface of maxillary and mandibular permanent first molars. The assessments were made for the established plaque and repeated for 48-hour-old plaque. Comparisons between plaque scores in partly and fully erupted teeth at both assessments showed a clear trend indicating that microbial growth conditions on occlusal surfaces were related to the degree in which the individual tooth was participating in oral function. Partly erupted teeth accumulated significantly higher amounts of plaque than fully erupted teeth $(\mathrm{p}<$ 
0.001). The detailed mapping showed that distribution and accumulation of dental plaque on occlusal surfaces were highly related to tooth-specific occlusal macromorphology.

Subsequently, hypothesis 3 tested the relationship between plaque accumulation and caries development on occlusal surfaces, as described below.

Hypothesis 3:

During Tooth Eruption, the Mechanical Oral Function

Is Reduced, Favoring Caries Initiation on Occlusal

Surfaces

The presence of active and inactive lesions at non-cavitated as well as cavitated levels on occlusal surfaces was recorded [Carvalho et al., 1989]. The locations of these lesions were mapped on morphology cards. Location and activity of caries lesions were highly correlated with occlusal plaque distribution and accumulation, which was in turn determined by mechanical function $(\mathrm{p}<0.010)$. On this basis, the influence of oral mechanical forces on occlusal plaque formation and caries development was verified. In order to further evaluate this assumption (hypothesis 4 ), a longitudinal study was required.

\section{Hypothesis 4:}

Intensive Mechanical Plaque Control during Tooth

Eruption Prevents Occlusal Caries Progression

A non-operative caries treatment was implemented based on a combination of educating children and their parents on home-based plaque control and professional plaque control [Carvalho et al., 1991]. Home-based plaque control during tooth eruption was a very important tool of the nonoperative treatment, particularly the self-performed/parental brushing of erupting teeth. Topical fluoride application was performed on non-cavitated active lesions, sealants were applied where non-cavitated active lesions were not arrested or had progressed at a non-cavitated level after 2-3 subsequent control visits, and fillings were placed when these previous treatments had failed and lesions had progressed to cavitation. Control visits were scheduled according to individual needs.

A control group with the same sociodemographic characteristics as the test group was formed based on examination of the records of children who were treated at the same child oral health care service [Christiansen, 2011]. Children in the control group received traditional preventive and restorative treatments which included a brushing instruction, regular topical fluoride application, systematic sealant application, fillings and regular visits according to individual needs.

Caries Process on Occlusal Surfaces
After 1 year, two examiners not involved in the treatment of the children carried out the examinations for plaque and caries. The results of the visible occlusal plaque index showed a significant reduction in plaque $(\mathrm{p} \leq$ 0.004). The results of the detailed mapping of occlusal plaque index highlighted that a majority of the anatomical sites of the groove-fossa system had a significant reduction in plaque $(\mathrm{p} \leq 0.05)$. Simultaneously, a significant reduction in the number of active non-cavitated lesions was observed. The evaluation of the lesions at individual anatomic sites showed whether the lesions had progressed, had not changed or had regressed. No changes and regression accounted for $80 \%$ of all observations. In the test group, fissure sealing was applied on 2 teeth, in contrast to 80 teeth in the control group. It was possible to control caries progression on emerging teeth by focused mechanical disturbance. Thus, hypotheses 3 and 4 were accepted. By taking this argument one step further, hypothesis 5 was tested.

\section{Hypothesis 5:}

Fully Erupted Teeth Require Less Intensive Control of Plaque to Prevent Caries Progression on Occlusal Surfaces

During a period of 3 years, the most dominant observation was the gradual decrease in plaque scores in all anatomical sites of the groove-fossa system [Carvalho et al., 1992]. Analyses of the individual enamel changes showed that $90 \%$ of the observations were related to no change and regression. This was mainly observed in the central and distal fossae of maxillary molars and in all anatomical sites of mandibular molars $(\mathrm{p} \leq 0.05)$. All teeth were radiographically recorded as sound. Moreover, less intensive mechanical plaque control measures were needed to control caries progression in fully erupted teeth over the study period. The need for control visits was reduced dramatically after the teeth had reached the last stage of eruption in comparison with the control group. Moreover, less professional resources were required compared with those previously used in connection with a traditional treatment strategy. In the test group, fissure sealing was applied to 10 teeth in contrast to 80 teeth in the control group. Accordingly, 7 teeth in the control group and 1 tooth in the test group were filled. It was therefore possible to accept hypothesis 5 .

These findings were complemented by testing hypothesis 6 on the influence of variations in sagittal molar occlusion on occlusal plaque formation and caries development in children. 
Hypothesis 6:

Variations in Sagittal Molar Occlusion Might

Interfere with the Growth Pattern of Occlusal Plaque

Formation and Caries Development on Fully Erupted

Permanent Molars

The clinical trial included children aged 7-10 years with permanent first molars which were clinically sound and at the final establishment of interdigitation [Ekstrand et al., 1993]. The results showed that maxillary molars with normal occlusion had the lowest number of sites with dental plaque and active caries $(\mathrm{p}<0.010)$. In addition, mandibular molars in normal and with one cusp distal occlusion had a lower number of sites with plaque and caries than all other occlusion types $(p<0.025)$. Thus, incomplete occlusal interdigitation before physiologic adjustment played a significant role in plaque accumulation, which was also related to occlusal caries activity.

Finally, the pattern of dental plaque formation, distribution and maturation within the dentition (hypothesis 7) was investigated.

\section{Hypothesis 7:}

The Formation, Distribution and Maturation of

Dental Plaque within the Dentition Follow a Pattern

Modulated by the Stage of Tooth Eruption

Caries-free children who were 4-6 years of age and with diastemata participated in the study [Carvalho et al., 2009a]. The children received prophylaxis and refrained from tooth brushing for $48 \mathrm{~h}$. The occurrence and distribution of dental plaque were assessed and pictures were taken. Limited amounts of plaque accumulated on fully erupted occlusal surfaces of primary molars in contrast to buccal surfaces $(\mathrm{p}<0.001)$. In the mixed and permanent dentitions, 48-hour plaque scores from the age of 6 up to 15 years were recorded, 3 times during eruption of the permanent first molars at the age of 6 years, then once when the permanent fist molars had fully erupted, and when the second molars had partly erupted at the age of 12 years. Finally, the last record was made when all permanent posterior teeth had fully erupted at the age of 15 years. At each visit, the observations were recorded photographically.

The difference in occlusal plaque accumulation between erupting first molars at the age of 6 years and fully erupted first molars at the age of 12 years was significant, with a marked reduction in thick plaque at the latter age. The same pattern was observed when occlusal plaque scores of permanent first molars in full occlusion were compared with those of emerging second molars. Finally, permanent first and second molars in full occlusion

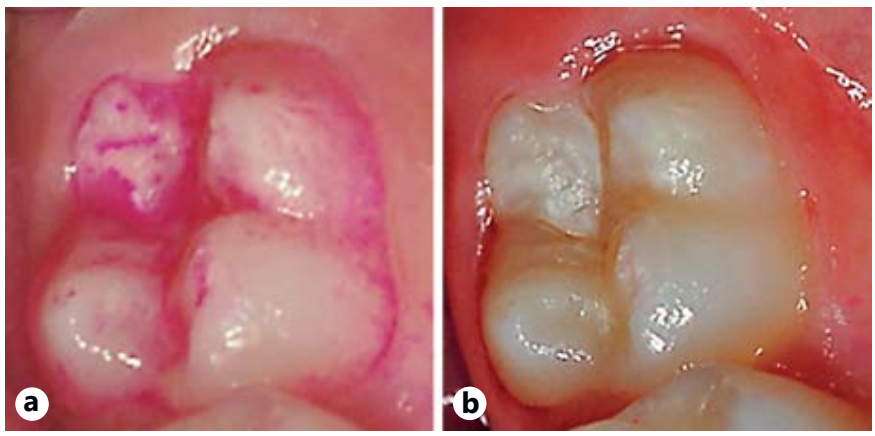

Fig. 1. Established plaque on erupting permanent first molar (a). The limited mechanical oral function of the erupting tooth offers good conditions for plaque accumulation and further development of active noncavitated occlusal caries lesions in the groovefossa system (b).

showed limited accumulation of thin plaque on occlusal surfaces at the age of 15 years. Thus, it was demonstrated that the pattern of plaque accumulation and distribution on occlusal surfaces was modulated by the stage of tooth eruption. On the other hand, the formation and distribution of thick plaque on approximal and free smooth surfaces did not show changes in relation to stage of tooth eruption.

Collectively, based on the results of these studies, it was possible to elucidate the biological principles determining the development and arrest of caries on occlusal surfaces. Clear evidence was provided to show that the most influential biological determinants of the development and arrest of occlusal caries were thick plaque accumulation on the groove-fossa system and the stage of tooth eruption limiting mechanical oral function, as illustrated in figure 1.

In a wider perspective, these principles were applied in subsequent clinical trials aiming at controlling caries progression in populations of children and adolescents with their permanent molars at different stages of eruption [Ekstrand et al., 2000; Maltz et al., 2003; Frazão, 2011; Vermaire, 2013]. These studies had different designs and conceptions of non-operative treatment programs, were carried out in dissimilar countries and over a period of time ranging from 18 months to 3 years. However, the results of these studies had common features among them and with those presented in this review.

The most common findings were related to a marked reduction in occlusal plaque scores in fully erupted teeth compared with erupting teeth. Also, significant differences were described between test and control groups regarding the occurrence of plaque and caries incidence on 
occlusal surfaces of permanent molars $(\mathrm{p}<0.05)$. In the studies by Ekstrand et al. [2000] and Maltz et al. [2003], caries incidence was measured at the non-cavitated level, and arrest of noncavitated active lesions was observed during the study period. A significant reduction in caries incidence, measured at the cavitated level, was observed in the test groups of the studies by Frazão [2011] and by Vermaire [2013]. All the studies observed that there was a marked reduction in the number of visits for control of caries progression in the test group after the molars had reached functional occlusion.

\section{Conclusion}

The relevance of the present review lies in the new understanding of and insight into biological determinants for caries development during tooth eruption and its management. The most influential biological determi- nants for caries development and arrest on occlusal surfaces are thick plaque accumulation on the groove-fossa system and the stage of tooth eruption.

The period of tooth eruption is considered a risk factor for caries development because occlusal surfaces offer good conditions for plaque accumulation and further caries development in this period due to their limited mechanical oral function. However, occlusal caries may be controlled by nonoperative measures if such measures are implemented from the very beginning of tooth eruption and maintained until the tooth is in full occlusion. State-of-the-art measures to control occlusal caries progression should be implemented from the very beginning of tooth eruption.

\section{Disclosure Statement}

The author has no conflict of interest to declare.

\section{References}

Aoba T, Fejerskov O: Dental fluorosis: chemistry and biology. Crit Rev Oral Biol Med 2002;13: 155-170.

Baelum V, Nyvad B, Gröndahl HG, Fejerskov O. The foundations of good diagnostic practice; in Fejerskov O, Kidd E (eds): Dental Caries: The Disease and Its Clinical Management, ed 2. Oxford, Blackwell Munksgaard, 2008, pp 103-118.

Carlsen O: Dental Morphology. Copenhagen, Munksgaard, 1987.

Carvalho JC, Ekstrand KR, Thylstrup A: Dental plaque and caries on occlusal surfaces of first permanent molars in relation to stage of eruption. J Dent Res 1989;68:773-779.

Carvalho JC, Ekstrand KR, Thylstrup A: Results after 1 year of non-operative occlusal caries treatment of erupting permanent first molars. Community Dent Oral Epidemiol 1991;19:23-28.

-Carvalho JC, Figueiredo CS, Mestrinho HD: Clinical report on plaque formation, distribution and maturation within the primary, mixed and permanent dentitions. Eur J Paediatr Dent 2009a;10:193-199.

Carvalho JC, Figueiredo MJ, Vieira EO, Mestrinho HD: Caries trends in Brazilian non-privileged preschool children in 1996 and 2006. Caries Res 2009b;43:2-9.

Carvalho JC, Silva EF, Fonseca JAC, Gomes RR, Mestrinho HD: Impact of enamel defects on early caries development in preschool children. Caries Res 2011;45:353-360.

Carvalho JC, Thylstrup A, Ekstrand KR: Results after 3 years of non-operative occlusal caries treatment of erupting permanent first molars. Community Dent Oral Epidemiol 1992;20: 187-192.

\footnotetext{
Carvalho JC, Van Nieuwenhuysen JP, D'Hoore $\mathrm{W}$ : The decline in dental caries among Belgian children between 1983 and 1998. Community Dent Oral Epidemiol 2001;29:55-61.

Carvalho JC, Van Nieuwenhuysen JP, Maltz M Traitement non-opératoire de la carie dentaire. Real Clin 2004;15:235-248.

Christiansen J: Non-operative caries treatment; in Splieth CH (ed): Revolutions in Pediatric Dentistry. Berlin, Quintessence, 2011, pp 21-35.

-Ekstrand KR, Bjørndal L: Structural analyses of plaque and caries in relation to the morphology of the groove-fossa system on erupting mandibular third molars. Caries Res 1997;31: 336-348.

Ekstrand KR, Kuzmina IN, Kuzmina E, Christiansen MEC: Two and a half-year outcome of caries-preventive programs offered to groups of children in the Solntsevsky district of Moscow. Caries Res 2000;34:8-19.

Fejerskov O, Thylstrup A, Larsen MJ: Rational use of fluoride in caries prevention: a concept based on possible cariostatic mechanisms. Acta Odontol Scand 1981;39:241-249.

-Ferreira Zandoná A, Santiago E, Eckert GJ, Katz BP, Pereira de Oliveira S, Capin OR, Mau M, Zero DT: The natural history of dental caries lesions: a 4-year observational study. J Dent Res 2012;91:841-846.

-Fontana M, Santiago E, Eckert GJ, Ferreira Zandoná AG: Risk factors of caries progression in a Hispanic school-aged population. J Dent Res 2011;90:1189-1196.

Frazão P: Effectiveness of the bucco-lingual technique within a school-based supervised toothbrushing program on preventing caries: a randomized controlled trial. BMC Oral Health 2011;11:11.

Hevinga M: Management of occlusal caries; $\mathrm{PhD}$ thesis, Radboud University Nijmegen, 2011.

Ekstrand KR, Nielsen LA, Carvalho JC, Thylstrup A: Dental plaque and caries on permanent first molar occlusal surfaces in relation to sagittal occlusion. Scand J Dent Res 1993;101:915.

Elfrink ME, ten Cate JM, Jaddoe VW, Hofman A, Moll HA, Veerkamp JS: Deciduous molar hypomineralization and molar incisor hypomineralization. J Dent Res 2012;91:551-555.

Fejerskov O: Changing paradigms in concepts on dental caries: consequences for oral health care. Caries Res 2004;38:182-191.

Fejerskov O, Thylstrup A: The oral environment: an introduction; in Thylstrup A, Fejerskov O (eds): Textbook of Clinical Cariology, ed 2. Copenhagen, Munksgaard, 1994, pp 13-16.

Ismail AI, Lim S, Sohn W, Willem JM: Predictors of dental caries progression in primary teeth. J Dent Res 2009;88:270-275.

-Kataoka S, Sakuma S, Wang J, Yoshihara A, Miyazaki $\mathrm{H}$ : Changes in electrical resistance of sound fissure enamel in first molars for 66 months from eruption. Caries Res 2007;41: 161-164.

Kotsanos N, Darling AI: Influence of posteruptive age of enamel on its susceptibility to artificial caries. Caries Res 1991;25:241-250.

Kühnisch J, Galler M, Seitz M, Stich H, Lussi A, Hickel R, Kunzelmann KH, Bücher K: Irregularities below the enamel-dentin junction may predispose for fissure caries. J Dent Res 2012;91:1066-1070.
} 
Liu BY, Lo EC, Chu CH, Lin HC: Randomized trial on fluorides and sealants for fissure caries prevention. J Dent Res 2012;91:753-758.

- Maltz M, Barbachan e Silva B, Carvalho DQ, Volkweis A: Results after two years of nonoperative treatment of occlusal surface in children with high caries prevalence. Braz Dent J 2003;14:48-54.

Marsh PD: Dental plaque as a microbial biofilm. Caries Res 2004;38:204-211.

Marthaler TM: Changes in dental caries 19532003. Caries Res 2004;38:173-181.

Mattila ML, Rautava P, Aromaa M, Ojanlatva A, Paunio P, Hyssälä L, Helenius H, Sillanpää M: Behavioural and demographic factors during early childhood and poor dental health at 10 years of age. Caries Res 2005;39:85-91.

- Mejàre I, Stenlund H, Zelezny-Holmlund C: Caries incidence and lesion progression from adolescence to young adulthood: a prospective 15 -year cohort study in Sweden. Caries Res 2004;38:130-141.

-Muller-Bolla M, Courson F, Droz D, Lupi-Pégurier L, Velly AM: Definition of at-risk occlusal surfaces of permanent molars-a descriptive study. J Clin Pediatr Dent 2009;34:35-42.

-Nyvad B: Diagnosis versus detection of caries. Caries Res 2004;38:192-198.
Pearce EIF, Larsen MJ, Coote GE: Fluoride in enamel lining pits and fissures of the occlusal groove-fossa system in human molar teeth. Caries Res 1999;33:196-205.

Schulte A, Gente M, Pieper K: Posteruptive changes of electrical resistance values in fissure enamel of premolars. Caries Res 1999;33: 242-247.

Skeie MS, Klock KS, Haugejorden O, Riordan PJ, Espelid I: Tracking of parents' attitudes to their children's oral health-related behavior Oslo, Norway, 2002-04. Acta Odontol Scand 2010;68:49-56.

Takahashi N, Nyvad B: Caries ecology revisited: microbial dynamics and the caries process. Caries Res 2008;42:409-418.

ten Cate JM: Contemporary perspective on the use of fluoride products in caries prevention. Br Dent J 2013;214:161-167.

ten Cate JM, Larsen MJ, Pearce EIF, Fejerskov O: Chemical Interactions between the tooth and oral fluids; in Fejerskov O, Kidd E (eds): Dental Caries: The Disease and Its Clinical Management, ed 2. Oxford, Blackwell Munksgaard, 2008, pp 209-230.

Tenuta LM, Cury JA: Fluoride: its role in dentistry. Braz Oral Res 2010;24(suppl 1):9-17.

Thylstrup A, Bruun C, Holmen L: In vivo caries models: mechanisms for caries initiation and arrestment. Adv Dent Res 1994;8:144-157.
Thylstrup A, Chironga L, Carvalho JC, Ekstrand KR: The occurrence of dental calculus in occlusal fissures as an indication of caries activity; in ten Cate JM (ed): Recent Advances in the Study of Dental Calculus. Oxford, IRL Press, 1989, pp 211-222.

Thylstrup A, Fejerskov O: Clinical and pathological features of dental caries; in Thylstrup A, Fejerskov O (eds): Textbook of Clinical Cariology, ed 2. Copenhagen, Munksgaard, 1994, pp 111-148.

Vermaire E: Optimizing oral health: towards a tailored, effective and cost-effective dental care; $\mathrm{PhD}$ thesis, ACTA, 2013.

-Wang J, Someya Y, Inaba D, Longbottom C, Miyazaki $\mathrm{H}$ : Relationship between electrical resistance measurements and microradiographic variables during remineralization of softened enamel lesions. Caries Res 2005;39:60-64.

Wang JD, Chen X, Frencken J, Du MQ, Chen Z: Dental caries and first permanent molar pit and fissure morphology in 7- to 8-year-old children in Wuhan, China. Int J Oral Sci 2012; 4:157-160.

-Watt RG: From victim blaming to upstream action: tackling the social determinants of oral health inequalities. Community Dent Oral Epidemiol 2007;35:1-11. 